\title{
Motion optimization of humanoid mobile robot with high redundancy
}

\author{
Hongxing Wang \\ State Key Laboratory of Robotics and System, Harbin Institute of Technology, Harbin, China and Robotics Institute, \\ School of Mechanical and Electrical Engineering, Nanchang Institute of Technology, Nanchang, China \\ LianZheng Ge, Ruifeng Li and Yunfeng Gao \\ State Key Laboratory of Robotics and System, Harbin Institute of Technology, Harbin, China, and \\ Chuqing Cao
}

Nanjing University of Science and Technology, Nanjing, China and of HIT Wuhu Robot Technology Research Institute of Harbin Institute of Technology, Wuhu, China

\begin{abstract}
Purpose - An optimal solution method based on 2-norm is proposed in this study to solve the inverse kinematics multiple-solution problem caused by a high redundancy. The current research also presents a motion optimization based on the 2-Norm of high-redundant mobile humanoid robots, in which a kinematic model is designed through the entire modeling.

Design/methodology/approach - The current study designs a highly redundant humanoid mobile robot with a differential mobile platform. The high-redundancy mobile humanoid robot consists of three modular parts (differential driving platform with two degrees of freedom (DOF), namely, left and right arms with seven DOF, respectively) and has total of 14 DOFs. Given the high redundancy of humanoid mobile robot, a kinematic model is designed through the entire modeling and an optimal solution extraction method based on 2-norm is proposed to solve the inverse kinematics multiple solutions problem. That is, the 2-norm of the angle difference before and after rotation is used as the shortest stroke index to select the optimal solution. The optimal solution of the inverse kinematics equation in the step is obtained by solving the minimum value of the objective function of a step. Through the step-by-step cycle in the entire tracking process, the kinematic optimization of the highly redundant humanoid robot in the entire tracking process is realized.

Findings - Compared with the before and after motion optimizations based on the 2-norm algorithm of the robot, its motion after optimization shows minimal fluctuation, improved smoothness, limited energy consumption and short path during the entire mobile tracking and operating process.

Research limitations/implications - In this paper, the whole kinematics model of the highly redundant humanoid mobile robot is established and its motion is optimized based on 2-norm, which provides a theoretical basis for the follow-up research of the service robot.

Practical implications - In this paper, the whole kinematics model of the highly redundant humanoid mobile robot is established and its motion is optimized based on 2-norm, which provides a theoretical basis for the follow-up research of the service robot.

Social implications - In this paper, the whole kinematics model of the highly redundant humanoid mobile robot is established and its motion is optimized based on 2-norm, which provides a theoretical basis for the follow-up research of the service robot.

Originality/value - Motion optimization based on the 2-norm of a highly redundant humanoid mobile robot with the entire modeling is performed on the basis of the entire modeling. This motion optimization can make the highly redundant humanoid mobile robot's motion path considerably short, minimize energy loss and shorten time. These researches provide a theoretical basis for the follow-up research of the service robot, including tracking and operating target, etc. Finally, the motion optimization algorithm is verified by the tracking and operating behaviors of the robot and an example.
\end{abstract}

Keywords Humanoid mobile robot, High redundancy, Entire modeling, 2-Norm, Motion optimization, 3D a*, humanoid robot

Paper type Research paper

\section{Introduction}

In recent years, the research direction of the mobile single-arm redundant robot (Han et al., 2017; Suryo Arifi and Hang, 2013; Liu et al., 2004) and the mobile dual-arm redundant robot has been the object of increasing attention. Research on the humanoid

The current issue and full text archive of this journal is available on Emerald Insight at: https://www.emerald.com/insight/0144-5154.htm

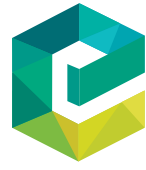

Assembly Automation

Emerald Publishing Limited [ISSN 0144-5154] [DOI 10.1108/AA-06-2020-0083]
(C) Hongxing Wang, LianZheng Ge, Ruifeng Li, Yunfeng Gao and Chuqing Cao. Published by Emerald Publishing Limited. This article is published under the Creative Commons Attribution (CC BY 4.0) licence. Anyone may reproduce, distribute, translate and create derivative works of this article (for both commercial and non-commercial purposes), subject to full attribution to the original publication and authors. The full terms of this licence maybe seen at http://creativecommons.org/licences/by/4.0/legalcode

This work was partially supported by Key Research and Development Program of Guangdong Province 2020B090928002, National Natural Science Foundation (NSFC) 61673136, National Natural Science Foundation (NSFC) 61763032, Research Project of Jiangxi Provincial Department of Education GJJ190941.

Conflict of Interests: The authors declare that there is no conflict of interests regarding the publication of this paper.

Received 20 April 2020

Revised 18 September 2020

21 October 2020

Accepted 20 November 2020 
mobile robot has mainly included the following aspects: according to the positive and inverse solutions on the kinematics of the humanoid dual-arm, the transformation matrix between the mobile robot and the arm simultaneously given (Ge et al., 2011). A manipulation planning method for both arms of a virtual human is proposed for product assembly and maintenance motion simulation under a complex virtual environment, a goal-oriented heuristic rapidly exploring random tree (RRT) algorithm is adopted with dual balance expansion and combined with the rapid arm inverse kinematical method (Wang and Li, 2009). Vahrenkamp (2009) studied the trajectory planning for a humanoid robot to grasp a plate without an inverse kinematical equation. Zhang and Ouyang (2013) and Li et al. (2009) focused on the path planning of a dual-robot-coordinated couple motion and proposed replacing the tool coordinate system of the slave robot for offline slave robot path generation. Sheng Jinhao focused on the selection of optimal inverse solution based on 2-norm, in which the 2-norm of every two degrees of freedom's (DOF) angular difference is used as the shortest stroke index(Sheng, 2017). Baratcart(2014) adopts 2-norm/infinity-norm switching resolution of biarticular actuation redundancy. It is shown that utilization of switching resolution improves both the motor size (with respect to 2-norm) and energy requirements (with respect to infinity-norm) of the system. The motion recovery methodologies are proposed to recover as many components of the manipulator twist as possible while minimizing the 2-norm of the joint velocity vector (Nazari and Notash, 2016). Xu et al. (2005) regards the manipulator and mobile platform as a series system and plans it online. Petersson et al. (2009) divides the system into two subsystems: mobile platform and manipulator for coordinated planning and control. However, these studies are limited because both model and motion optimization are for a single-arm robot. Overall, these studies lack information on the entire modeling and on the redundancy comparisons and on the motion optimization of highly redundant humanoid mobile robots.

Given the drawbacks of previous studies and the characteristics of highly redundancy of humanoid mobile robots, the current study proposes a whole modeling and motion optimization based on 2-norm of the robot. According to the comparative study between before and after motion optimizations of mobile robots with high redundancy, the root mean square error (RMSE) value of every DOF of the optimized model becomes smaller and has low volatility during the entire mobile tracking and operating process. Through simulation and comparative analysis, a highly redundant humanoid mobile robot optimized based on 2-norm shows superior motion performance, improved smoothness and considerable flexibility.

The remainder of this paper is organized as follows. Section 2 presents the motion optimization based on the 2-norm of a mobile humanoid robot. Section 3 conducts simulation and analysis before and after the robot motion optimization based on 2-norm. Section 4 uses an optimal path obtained by $\mathrm{A}^{*}$ search algorithm to verify the motion optimization based on the 2-norm algorithm of the robot (Zhu et al., 2007). Finally, Section 5 presents the conclusions and discussions between before- and after- optimization based on the 2-norm algorithm of the mobile humanoid robot with whole modeling.

\section{Motion optimization based on 2-norm on robot with whole kinematic model}

A whole modeling on a highly redundant humanoid mobile robot based on the whole resolved motion rate control (WRMRC) algorithm was built (Wang et al., 2016). Figure 1 shows the combination of the kinematic model of the differential driving platforms and fixed humanoid robot comprises the whole system kinematic model of a highly redundant humanoid mobile robot. Where $P_{s}$ in the kinematic model of the humanoid mobile robot represents the fixed intersection point of the first link and platform and its coordinates are $\left(x_{s}, y_{s}, 0\right)$ in Figure $1 ; P_{e}$ and $P_{e}^{\prime}$ are the left and right-arm end effectors, respectively, of the fixed humanoid robot; and their coordinates are $\left(x_{e}, y_{e}, z_{e}\right)$ and $\left(x_{e}^{\prime}, y_{e}^{\prime}, z_{e}^{\prime}\right)$, respectively; $l_{i}(i=1, \cdots, 4)$ is the left or right-arm length of the ith link; $\theta_{i}$ and $\theta^{\prime}{ }_{i}(i=1, \cdots, 7)$ are the left and right-arm ith joint angle, respectively, of the robot. The motion of the 1st, $3 \mathrm{rd}, 5$ th and 7 th joints are the rotary motion; and the motion of the 2 nd, 4 th and 6 th joints are the revolute motion.

A whole modeling on a highly redundant humanoid mobile robot based on the WRMRC algorithm can be obtained through the transformation from $\boldsymbol{\theta}_{d_{-}} m=\left[\boldsymbol{\theta}^{T}{ }_{w} \boldsymbol{\theta}_{d_{-}}^{T} l \boldsymbol{\theta}^{T}{ }_{d_{-}} r\right]^{T}$ to $\mathbf{P}_{e_{-}} d=\left[\mathbf{P}^{T}{ }_{s} \mathbf{P}^{T} e_{-} d_{-} l \mathbf{P}^{T} e_{e_{-}} d_{-} r\right]^{T}$ by the following homogenous transformation matrix [equation(1)]:

$$
\mathbf{P}_{e_{-}} d=R_{d \_m} \mathcal{F}_{d \_m} \boldsymbol{\theta}_{d_{-}} m
$$

where $\mathbf{P}_{e_{-}} d$ in equation $(1)$ is the $(8 \times 1)$ vector; $\mathbf{P}_{s}$ is the $(3 \times 1)$ vector; $\mathbf{P}_{e_{-}} d_{-} l$ and $\mathbf{P}_{e_{-}} d_{-} l$ are the $(3 \times 1)$ vector that denotes the left- and right-arm velocities, respectively, of the humanoid robot; $\boldsymbol{\theta}_{d_{-}} m$ is the $(14 \times 1)$ vector; $\boldsymbol{\theta}_{w}=\left[\theta_{r} \theta_{l}\right]^{T}$ is the $(2 \times 1)$ vector that denote the angular velocities of the left-wheel and right-wheel of this platform, respectively; $\boldsymbol{\theta}_{d_{-}} l$ and $\boldsymbol{\theta}_{d_{-}} r$ are the $(6 \times 1)$ vector that denote the angular velocities of the six left- and right-arm joints, respectively, of this robot; $R_{d \_m}$ is a $(8 \times 8)$ non-constant matrix related to $\phi$ for simplicity sake of the Generalized Jacobian Matrix (GJM) of the high redundant humanoid mobile robot; and $\mathscr{f}_{d_{-} m}$ is the $(8 \times 14)$ simplified GJM of the robot.

Given the high redundancy of the humanoid mobile robot, an optimal solution extraction method based on 2-norm is proposed to solve the inverse kinematics multiple solutions problem. That is, the 2-norm of the angle difference before and after rotation is used as the shortest stroke index to select the optimal solution. The high redundancy mobile humanoid robot consists of three modular parts: differential driving platform with two DOF, left and right arms with seven DOF, and a total of 14 DOFs. Every DOF's angles of the current and

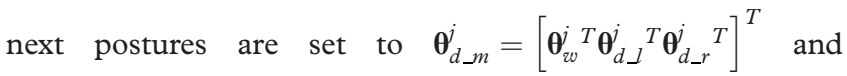
$\boldsymbol{\theta}_{d_{-} m}^{j+1}=\left[\left(\boldsymbol{\theta}_{w}^{j+1}\right)^{T}\left(\boldsymbol{\theta}_{d_{-l} l}^{j+1}\right)^{T}\left(\boldsymbol{\theta}_{d_{-} r}^{j+1}\right)^{T}\right]^{T}, \quad$ respectively.

The objective function is designed as follows:

$$
F_{j}=\left\|A_{\mathrm{j}}\right\|_{2}=\sqrt{\sum_{d \_m=1}^{14}\left(\boldsymbol{\theta}_{d \_m}^{j+1}-\boldsymbol{\theta}_{d \_m}^{j}\right)^{2}}
$$

Every DOF is set where $\theta_{l}$ and $\theta_{r}$ of $\boldsymbol{\theta}_{w}$ are the Nos. 1 and 2 $\mathrm{DOF}$, respectively, of $\boldsymbol{\theta}_{d \_m} ; \boldsymbol{\theta}_{d \_l}$ of $\boldsymbol{\theta}_{d \_m}$ are the Nos. 3 to $8 \mathrm{DOF}$ 
Figure 1 Humanoid mobile robot with 7 DOF per arm and its kinematic model

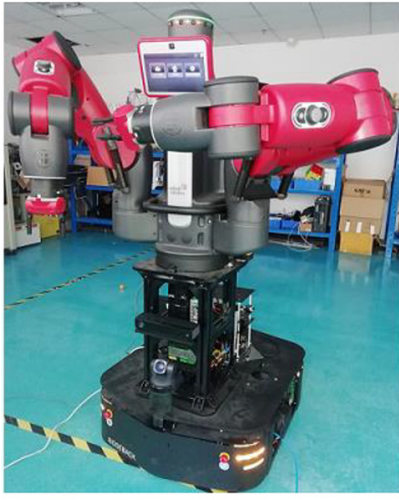

(a)

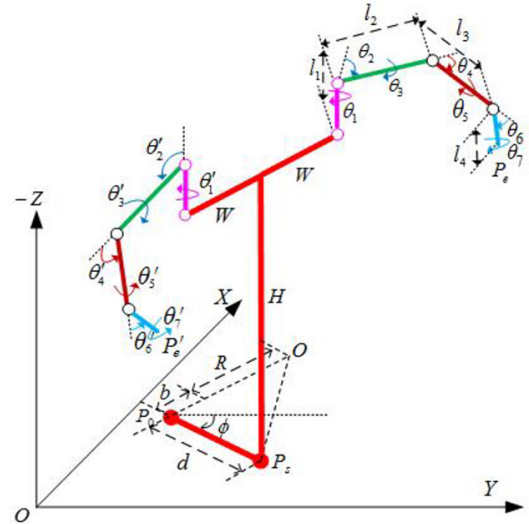

(b)

Notes: (a) Humanoid mobile robot with 7 DOF per arm; (b) kinematic model of the humanoid mobile robot with 7 DOF per arm

of $\boldsymbol{\theta}_{d \_m}$, respectively; and $\boldsymbol{\theta}_{d \_r}$ of $\boldsymbol{\theta}_{d \_m}$ are the Nos. 9 to 14 DOF, respectively, of $\boldsymbol{\theta}_{d_{-} m}$.

By solving the minimum value of the objective function of a step $j$, the optimal solution of the inverse kinematics equation in this step is obtained. Through the step-by-step cycle in the whole tracking process, the kinematic optimization of the highly redundant humanoid robot in the whole tracking process is realized. After the motion optimization based on the 2-norm, $\boldsymbol{\theta}_{d-m}=\left[\boldsymbol{\theta}_{w}^{T} \boldsymbol{\theta}_{d \_l}^{T} \boldsymbol{\theta}_{d}^{T} r\right]^{T}$

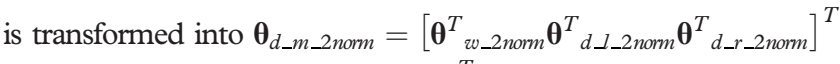
and $\quad \mathbf{P}_{e_{-} d}=\left[\mathbf{P}^{T}{ }_{s} \mathbf{P}^{T}{ }_{e_{-} d_{-} l} \mathbf{P}^{T}{ }_{e_{-} d_{-} r}\right]^{T}$ is transformed into $\mathbf{P}_{e_{-} d_{-} 2 \text { norm }}=\left[\mathbf{P}^{T}{ }_{s-2 n o r m} \mathbf{P}^{T}{ }_{e_{-} d_{-} l_{-} 2 n o r m} \mathbf{P}^{T}{ }_{e_{-} d_{-} r_{-} 2 n o r m}\right]^{T}$. The position relationship between $\boldsymbol{\theta}_{d \_} \_2$ norm and $\mathbf{P}_{e \_d \_2 n o r m}$ can be described by equation (3):

$$
\mathbf{P}_{e \_d \_2 n o r m}=f\left(\boldsymbol{\theta}_{d \_m \_2 n o r m}\right)
$$

where $\mathbf{P}_{e \_d}$ and $\mathbf{P}_{e \_d \_2 n o r m}$ are the $(8 \times 1)$ vectors; $\boldsymbol{\theta}_{d \_m \_2 n o r m}$ is the $(14 \times 1)$ vector; $\mathbf{P}_{\mathbf{s} \_2 n o r m}, \mathbf{P}_{e \_d \_l \_ \text {norm }}$, and $\mathbf{P}_{e \_d \_r \_2 n o r m}$ are the corresponding positions optimized based on the 2-norm of $P_{s}, P_{e}$ and $P_{e}^{\prime}$, respectively; and $\boldsymbol{\theta}_{w \_2 n o r m}, \boldsymbol{\theta}_{d \_l \_2 n o r m}$, and $\boldsymbol{\theta}_{d \_r} \_$2norm are the corresponding angles optimized based on the 2-norm of $\theta_{l}, \theta_{r}, \theta_{i}$ and $\theta_{i}^{\prime}(i=1, \cdots, 6)$, respectively.

For convenience of simulation, equation (3) is described as follows in the form of an angle variation in unit time:

$$
\Delta \boldsymbol{\theta}_{d \_m \_2 n o r m}=\mathcal{F}^{-1}{ }_{d \_m \_2 n o r m} R^{-1}{ }_{d \_m \_2 n o r m} \Delta \mathbf{P}_{e_{-} d \_2 n o r m}
$$

Where $\Delta \boldsymbol{\theta}_{d \_m \_2 n o r m}$ is the $(14 \times 1)$ vector; $\Delta \mathbf{P}_{e \_d \_2 n o r m}=$

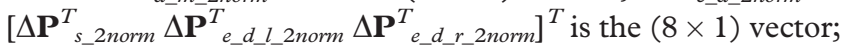
$\Delta \theta_{l \_2 n o r m}$ and $\Delta \theta_{r_{-} 2 n o r m}$ of $\Delta \boldsymbol{\theta}_{w_{\_} 2 n o r m}, \Delta \boldsymbol{\theta}_{d \_l \_2 n o r m}$, and $\Delta \boldsymbol{\theta}_{d \_r \_2 n o r m}$ are the corresponding angular variations optimized based on the 2-norm per unit time of $\theta_{l}, \theta_{r}, \theta_{i}$ and $\theta_{i}^{\prime}(i=1, \cdots, 6)$, respectively; and $\Delta \mathbf{P}_{s \_2 n o r m}, \Delta \mathbf{P}_{e \_d \_l \_n o r m}$, and $\Delta \mathbf{P}_{e_{-} d \_r \_2 n o r m}$ are the corresponding position variation optimized based on the 2norm per unit time of $P_{s}, P_{e}$ and $P_{e}^{\prime}$, respectively.

The specific processes in the simulation are listed as follows:
- Input the initial configuration of the highly redundant humanoid mobile robot, including the differential driving platform and dual arms.

- Input the different trajectories of the dual-arm end effectors of the mobile humanoid robot.

- As the unit time $\Delta T$ is confirmed, the whole steps $N$ and corresponding $\Delta \mathbf{P}_{e \_d \_ \text {norm }}$ are also confirmed.

- $\Delta \boldsymbol{\theta}_{d \_m \_2 n o r m}$ is calculated using equation (4).

- According to the value of $\Delta \boldsymbol{\theta}_{d \_m \_2 n o r m}$, the movements of optimization $\boldsymbol{\theta}_{d \_m}^{j+1}=\left[\boldsymbol{\theta}_{w}^{j+1}{ }^{+1} \boldsymbol{\theta}_{d \_l}^{j+1}{ }^{T} \boldsymbol{\theta}_{d \_r}^{j+1} T\right]^{T}$ zed $\Delta \boldsymbol{\theta}_{w \_2 n o r m}$, $\Delta \boldsymbol{\theta}_{d \_l \_2 n o r m}$ and $\Delta \boldsymbol{\theta}_{d_{-} r_{-} 2 \text { norm }}$ based on 2-norm are given in order.

- The optimized next motion status of the mobile humanoid robot is confirmed. If the number of whole steps is less than $N$, then the program will go to $\Delta \boldsymbol{\theta}_{d \_m \_2 n o r m}$ again. Otherwise, it will go to End.

- Comparison of the simulation results between before and after optimizations based on the 2-norm algorithm of the mobile humanoid robot. Comparison of the $\Delta \boldsymbol{\theta}$ and $\Delta \boldsymbol{\theta}_{w \_2 n o r m}, \Delta \boldsymbol{\theta}_{d \_l}$ and $\Delta \boldsymbol{\theta}_{d \_l \_2 n o r m}, \Delta \boldsymbol{\theta}_{d \_r}$ and $\Delta \boldsymbol{\theta}_{d \_r}{ }_{-2 n o r m}$ separately as list.

- End algorithm.

\section{Simulations and comparison before and after motion optimization}

The simulation of motion optimization based on the 2-norm of the redundant humanoid mobile robot with whole modeling is conducted. Compared with the before and after motion optimizations of the mobile humanoid robot, the optimized RMSE values of every DOF and every step of the mobile humanoid robot decrease, and the high redundancy mobile humanoid robot shows superior motion performance. The optimized path is shortened, thereby saving the whole tracking operation time. These advantages and effectiveness of the 
Figure 2 Movement simulations of dual-arm tracking different targets of humanoid robot with whole modeling before and after motion optimizations based on 2-norm in 3D

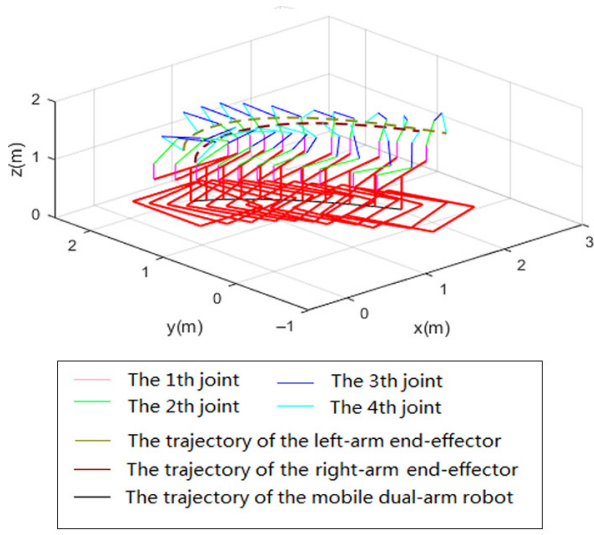

(a)

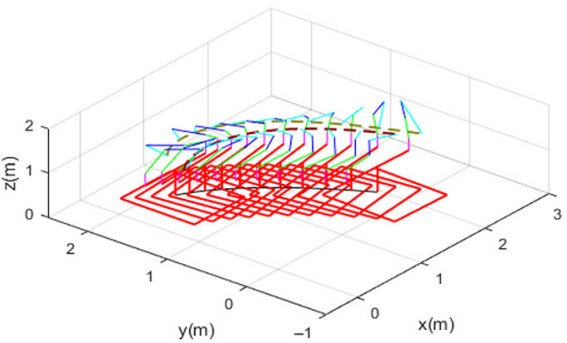

The 1th joint — The 3th joint
The 2th joint - The 4th joint
The trajectory of the left-arm end-effector
— The trajectory of the right-arm end-effector
T The trajectory of the mobile dual-arm robot

(b)

Notes: (a) Simulation of the dual-arm tracking different targets in 3D; (b) simulation of the motion optimization based on 2-norm in 3D

Figure 3 Angular variation comparison of 14 DOF in dual-arm tracking different targets of humanoid robot before and after motion optimizations
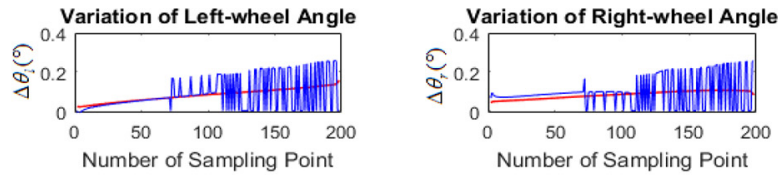

(a)
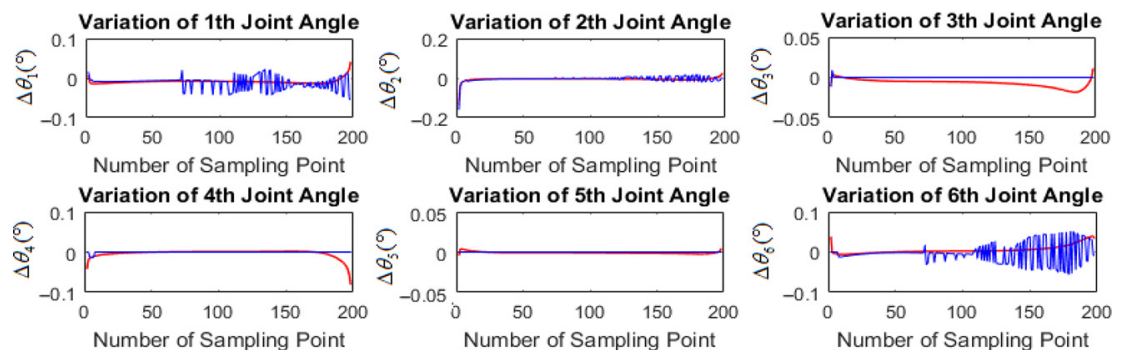

(b)
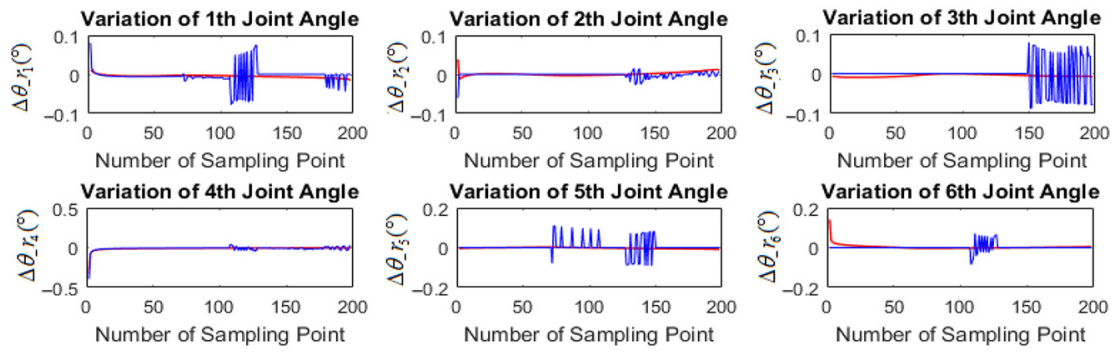

(c)

Notes: (a) Angular variation comparison of the dual-wheel; (b) six joint angular variations comparison of the left arm; (c) six joint angular variation comparison of right arm (Blue line indicates the angular variations before motion optimization; red line indicates the angular variations after motion optimization; the same below) 
Figure 4 RMSE value comparison of the angular variations of 14 DOF at each step in the dual-arm tracking different target processing before and after optimization
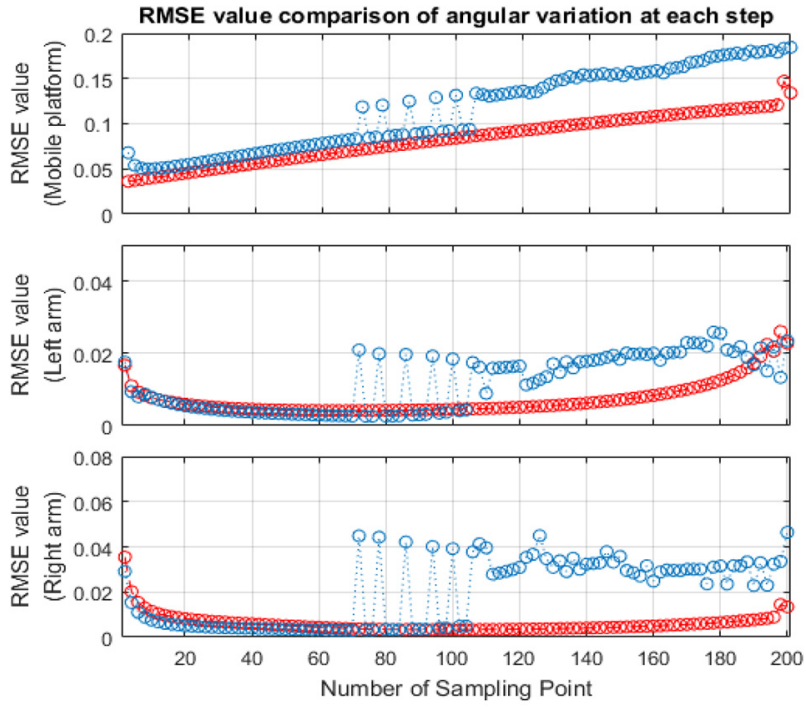

RMSE value of angular variation before optimization RMSE value of angular variation after optimization motion optimization algorithm is verified by the tracking and operating behaviors of the robot, respectively.

3.1 Simulations of motion optimization based on the 2norm of dual-arm tracking different targets of humanoid robot based on kinematic model of WRMRC algorithm

The simulations before and after motion optimizations based on the 2-norm algorithm are shown in Figure 2.

By comparing the simulation results before and after motion optimizations based on 2-norm, the angular variation comparison diagram of 14 DOFs of the high redundant mobile robot can be obtained, as shown in Figure 3. The 14 DOFs are composed of 2 DOFs for the left and right wheels of the mobile platform, as shown in Figure 3(a); and 6 DOFs for the left and right arms of the robot, respectively, as shown in Figures 3(b) and 3(c).

Through comparative analysis, the angular variations of 14 DOFs of highly redundant humanoid mobile robot after motion optimization based on 2-norm are smaller, with minimal fluctuation and improved smoothness in the whole tracking process. In particular, the optimization effects of the left and right wheel angular variations of the mobile platform are considerably evident, as shown in Figure 3(a). The validity of motion optimization has been completely proven by the simulation experiment of the dual arm tracking the different

Table 1 RMSE Value comparison of the angular variations of 14 DOFs in dual-arm tracking different target processing before and after optimization

\begin{tabular}{|c|c|c|c|c|c|c|c|c|c|c|c|c|c|c|}
\hline \multirow[b]{2}{*}{14 DOFs } & \multicolumn{2}{|c|}{ Mobile platform } & \multicolumn{6}{|c|}{ Left arm } & \multicolumn{6}{|c|}{ Right arm } \\
\hline & Left wheel & Right wheel & 1 & 2 & 3 & 4 & 5 & 6 & 1 & 2 & 3 & 4 & 5 & 6 \\
\hline $\begin{array}{l}\text { RMSE values } \\
\text { RMSE values }\end{array}$ & 0.1201 & 0.1261 & 0.0207 & 0.0148 & 0.0011 & 0.0018 & 0.0000 & 0.0248 & 0.0236 & 0.0069 & 0.0360 & 0.0320 & 0.0332 & 0.0160 \\
\hline (Optimized) & 0.0901 & 0.0836 & 0.0168 & 0.0118 & 0.0108 & 0.0113 & 0.0021 & 0.0115 & 0.0067 & 0.0052 & 0.0060 & 0.0255 & 0.0040 & 0.0119 \\
\hline
\end{tabular}

Figure 5 Movement simulations of the dual-arm operating different targets of humanoid robot with whole modeling before and after motion optimizations based on 2-norm in $3 \mathrm{D}$

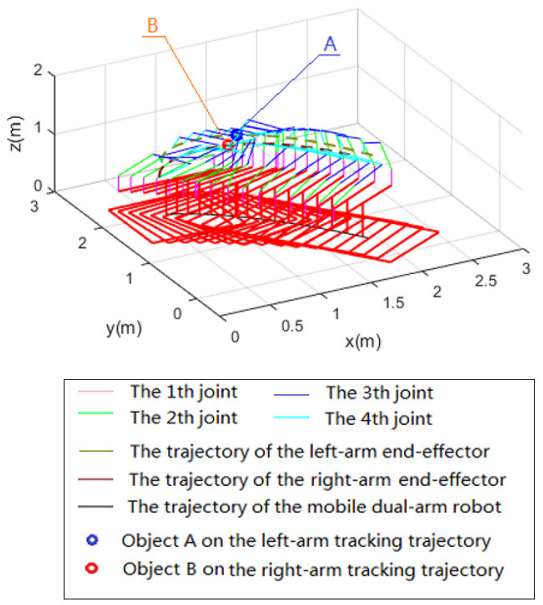

(a)

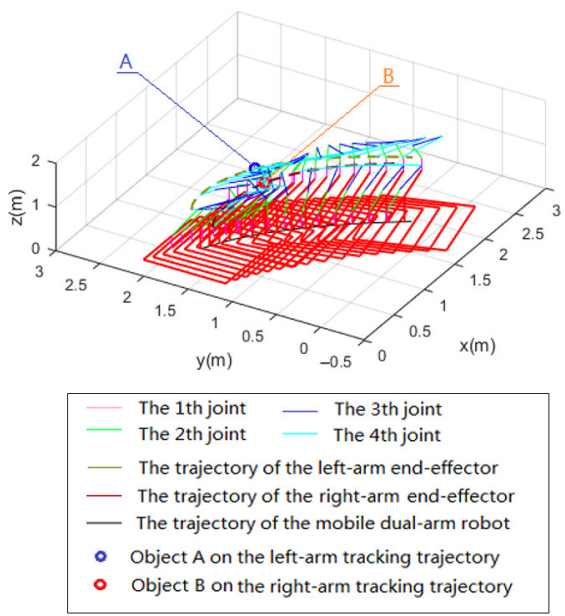

(b)

Notes: (a) Simulation of the dual-arm operating different targets in 3D; (b) simulation of motion optimization based on 2-norm in 3D 
Figure 6 Angular variation comparison of 14 DOFs in dual-arm mobile operating on static Object A for the left arm and on static Object B of the humanoid robot before and after motion optimization based on 2-norm with whole modeling
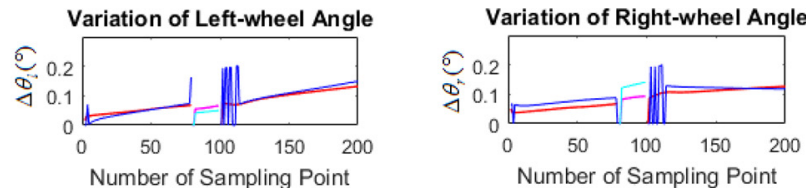

The variation of the joint angle in the trace

The variation of the joint angle in operation

(a)
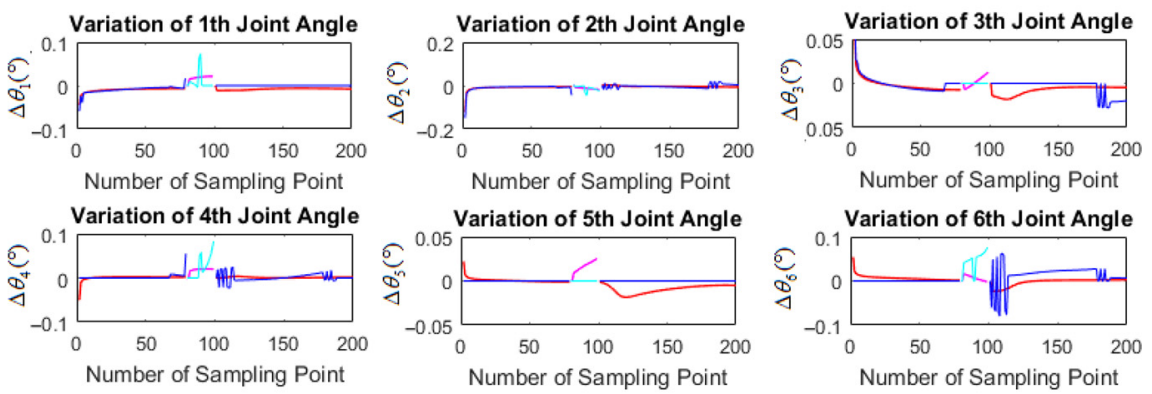

The variation of the joint angle in the trace

(b)
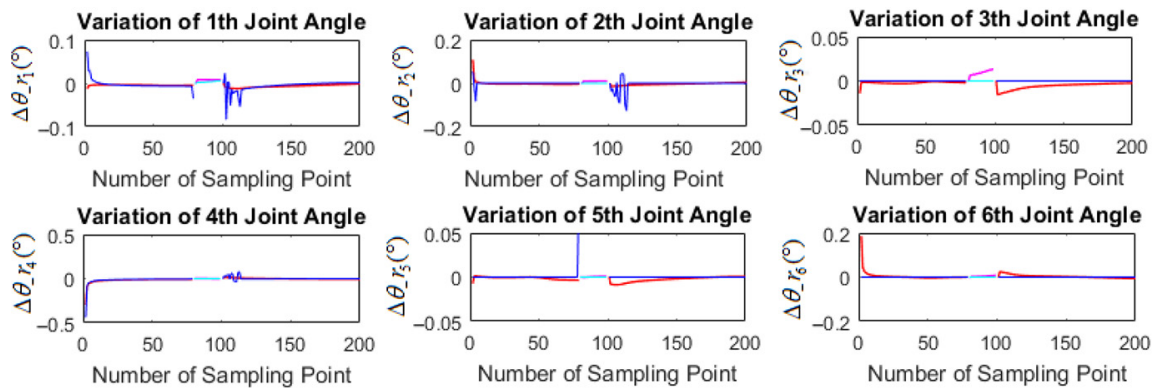

The variation of the joint angle in the trace

The variation of the joint angle in operation

(c)

Notes: (a) Angular variation comparison of the dual-wheel; (b) six joint angular variation comparison of the left arm; (c) six joint angular variation comparison of the right arm

targets of the highly redundant humanoid mobile robot with whole modeling.

Intuitively, the robot is divided into three parts to compare and analyze the MRSE value at each step in the tracking process, as shown in Figure 4. The three parts are the left and right wheels of the mobile platform and 6 DOFs of the left and right arms of the robot (same below).

Each optimized RMSE value of 14 DOFs is also generally lower than the non-optimized values during the dual-arm tracking different targets processing. This result further proves the effectiveness of motion optimization based on 2-norm from the perspective of energy reduction, as shown in Table 1.
On the basis of whole kinematic model of the WRMRC algorithm, the motion of the highly redundant humanoid mobile robot by motion optimization based on 2-norm has slight fluctuation, improved smoother, minimal energy consumption and short path. The validity of the algorithm has been proven in many aspects.

3.2 Simulations of motion optimization based on the 2norm of dual-arm mobile operating of humanoid robot with whole modeling

The simulations before and after motion optimization based on the 2-norm algorithm were conducted to verify the 
manipulability of the highly redundant humanoid mobile robot on different targets. These targets are Object A, which is an operation point on the end-effector trajectory of the left arm; and Object $\mathrm{B}$, which is an operation point on the end-effector trajectory of the right arm, as shown in Figure 5.

By comparing the simulation results before and after motion optimization based on 2-norm, the angular variation comparison diagram of 14 DOFs of a highly redundant mobile robot can be obtained, as shown in Figure 6. This robot includes $2 \mathrm{DOF}$ for the left and right wheels of the mobile platform, as shown in Figure 6(a) and 6 DOFs for the left and right arms of the robot, as shown in Figures 6(b) and 6(c), respectively.

Through the comparative analysis shown in Figure 6, the validity of motion optimization has been further proven by the simulation experiment of the dual arm operating the different targets of the highly redundant humanoid mobile robot with whole modeling.

Similarly, the robot is also divided into three parts to compare and analyze the MRSE values at each step in tracking different target processes, as shown in Figure 7. Through the analysis and comparison of the three tobot parts, the conclusion

Figure 7 RMSE value comparison of the angular variations of 14 DOFs at each step in the dual-arm mobile operating on static Object $A$ for the left arm and on static Object B before and after optimization
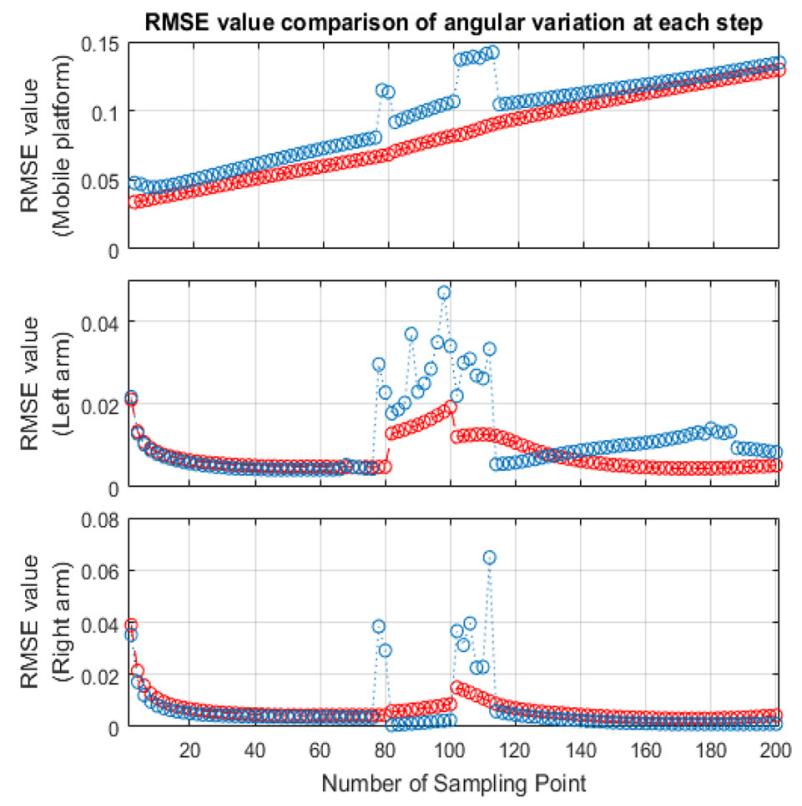

RMSE value of angular variation before optimization RMSE value of angular variation after optimization is the same because the robot tracks the same target in subsection 3.1 and the validity of motion optimization has been further proven.

Each optimized RMSE value of the 14 DOFs is also generally lower than the non-optimized value during the dual-arm operating different targets processing. This result further proves the effectiveness of the motion optimization based on 2-norm from the perspective of energy reduction, as shown in Table 2.

Evidently, the conclusion of the highly redundant humanoid mobile robot operating different targets after optimization is the same as that of the tracking the target(s). That is, the motion by optimization based on 2-norm has slight fluctuation, improved smoothness, minimal energy consumption and considerably short path. The validity of the algorithm has been proven in many aspects.

\section{Example verification}

\section{1 $3 \mathrm{D}$ environment construction and path optimization by $\mathbf{a}^{*}$ algorithm}

To further prove the effectiveness of the motion optimization based on 2-norm, the algorithm is verified by an example. First, a 3D map with obstacles is constructed. Second, an optimized path is found by using the $\mathrm{A}^{*}$ search algorithm in the map. Third, the WRMRC algorithm is used to track the optimized path. Accordingly, the motion is optimized by 2-norm. The effectiveness of the algorithm is proven by comparing the before and after optimizations. The optimized path is shown in Figure 8. where the blue point in optimization path chart is the starting point; and its coordinates are $(8,41,26)$. The green point is the terminal point; and its coordinates are $(45,4,41)$ as shown in Figure 8. In total, 26,614 points are searched from starting point

Figure 8 Optimized path by 3D A* heuristic algorithm

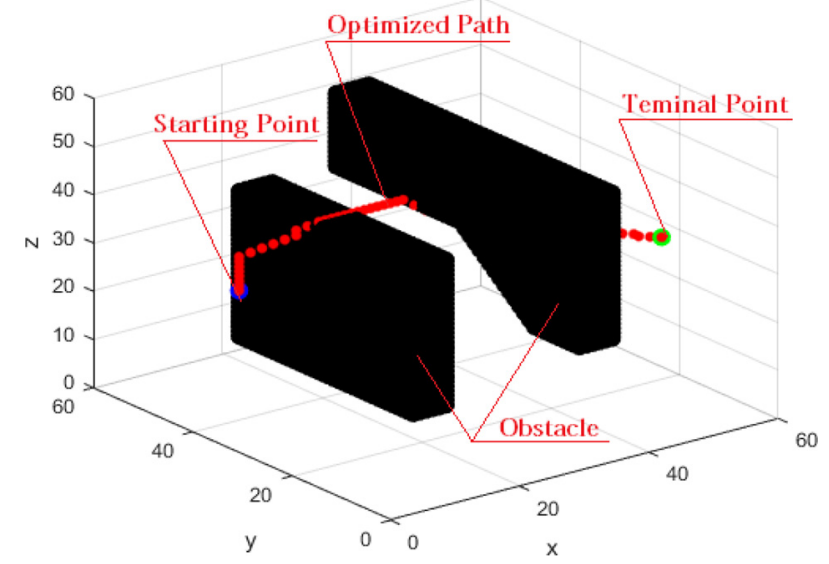

Table 2 RMSE value comparison of the angular variations of the 14 DOFs in dual-arm tracking different targets processing before and after optimization

\begin{tabular}{|c|c|c|c|c|c|c|c|c|c|c|c|c|c|c|}
\hline \multirow[b]{2}{*}{14 DOFs } & \multicolumn{2}{|c|}{ Mobile platform } & \multicolumn{6}{|c|}{ Left arm } & \multicolumn{6}{|c|}{ Right arm } \\
\hline & Left wheel & Right wheel & 1 & 2 & 3 & 4 & 5 & 6 & 1 & 2 & 3 & 4 & 5 & 6 \\
\hline $\begin{array}{l}\text { RMSE value } \\
\text { RMSE value }\end{array}$ & 0.0935 & 0.1072 & 0.0121 & 0.0155 & 0.0105 & 0.0141 & 0.0060 & 0.0257 & 0.0124 & 0.0180 & 0.0000 & 0.0345 & 0.0098 & 0.0000 \\
\hline (Optimized) & 0.0830 & 0.0918 & 0.0109 & 0.0116 & 0.0088 & 0.0074 & 0.0096 & 0.0087 & 0.0064 & 0.0096 & 0.0059 & 0.0244 & 0.0029 & 0.0150 \\
\hline
\end{tabular}


Figure 9 Movement simulations of the dual-arm tracking optimized path by the 3D $A^{*}$ heuristic algorithm of humanoid robot with whole modeling before and after motion optimization based on 2-norm in 3D

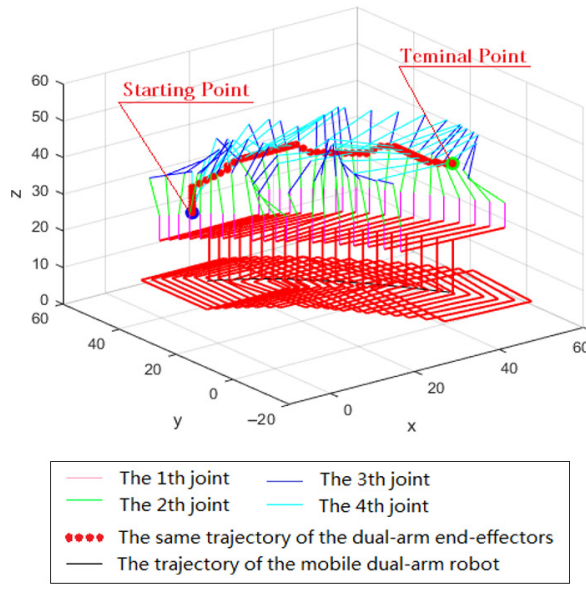

(a)

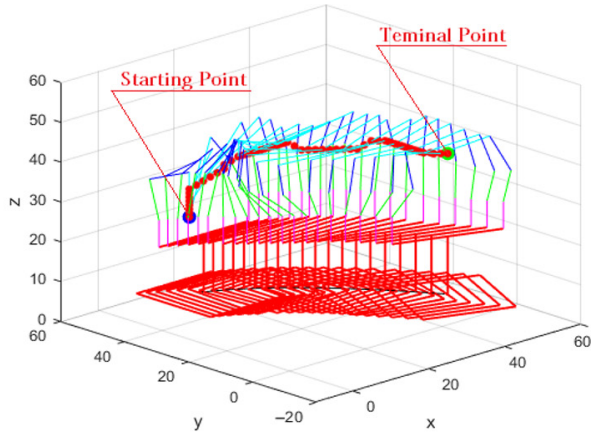

The 1th joint — The 3th joint

The 2th joint The 4th joint

..... The same trajectory of the dual-arm end-effectors

- The trajectory of the mobile dual-arm robot

Notes: (a) Simulation of the dual-arm tracking optimized path in 3D; (b) simulation of the motion optimization based on 2-norm in $3 \mathrm{D}$

Figure 10 Angular variation comparison of the 14 DOFs in the dual-arm tracking same optimized path by $3 \mathrm{D}^{*}$ heuristic algorithm of the humanoid robot before and after motion optimization based on 2-norm with whole modeling
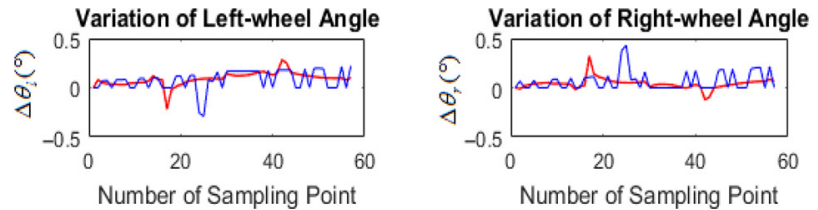

(a)
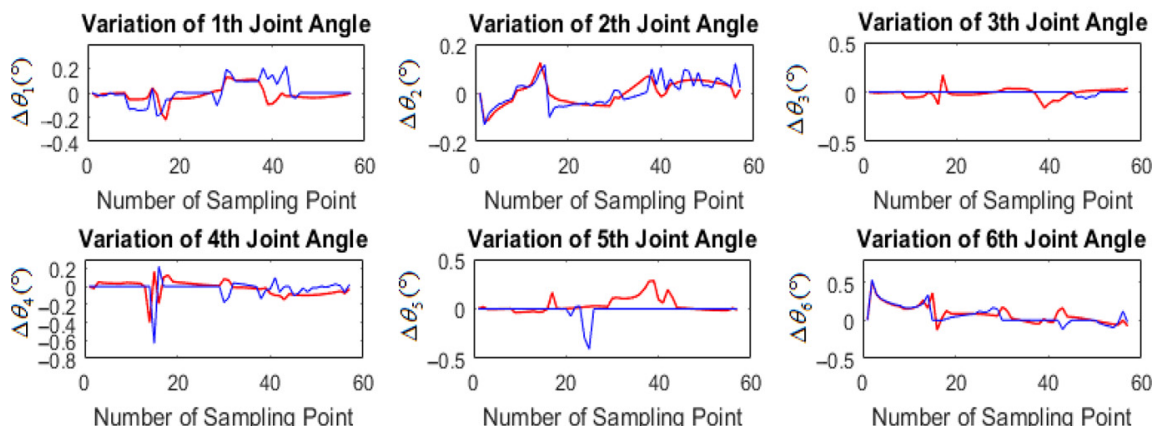

(b)
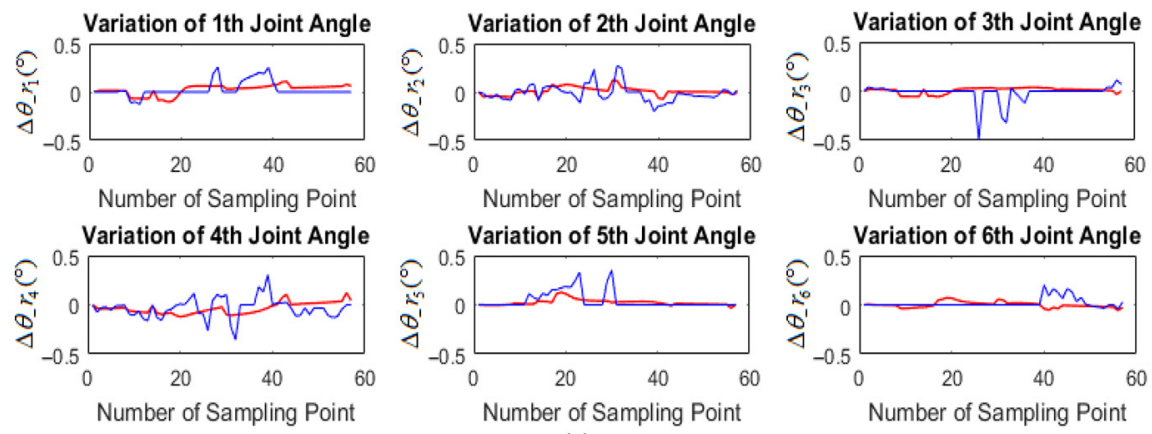

(c)

Notes: (a) Angular variation comparison of the dual-wheel; (b) six joint angular variation comparison of the left arm; (c) six joint angular variation comparison of the right arm 
Figure 11 RMSE value comparison of the angular variations of the 14 DOF at each step in the dual-arm tracking the same optimized path by $3 \mathrm{D} \mathrm{A}^{*}$ heuristic algorithm before and after optimization
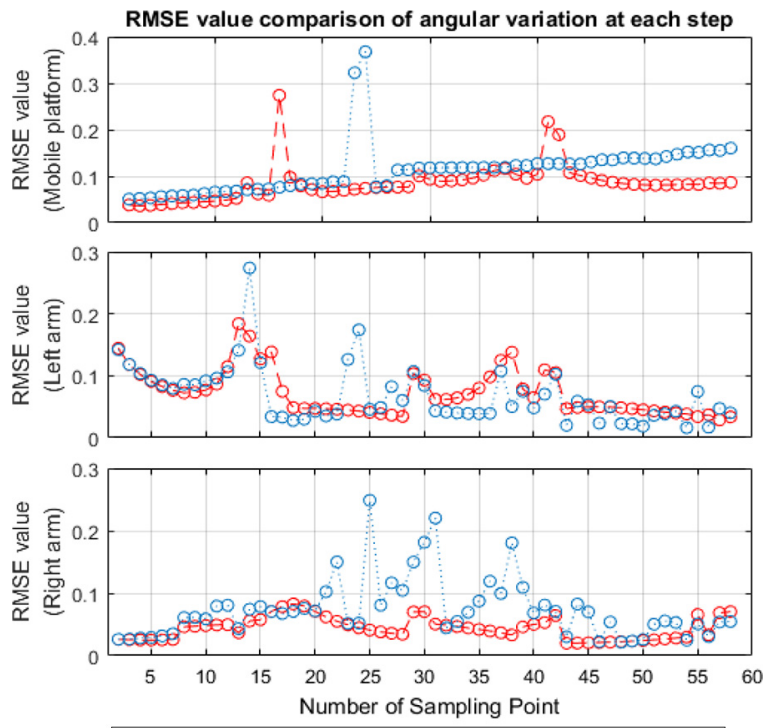

RMSE value of angular variation before optimization RMSE value of angular variation after optimization

to terminal point. It takes 59 steps during the tracking process, which takes $155.50 \mathrm{~s}$. The path length is 71.44 and the total cost is $2.86 \mathrm{~m}$ because the robot is magnified 25 times in the simulation.

\subsection{Simulation of the dual-arm tracking the optimized path obtained by the $a^{*}$ algorithm of humanoid robot with motion optimization based on 2 -norm}

Without considering the mobile platform and all joint obstacle avoidance, the numerical simulations before and after motion optimization based on the 2-norm of dual-arm tracking the optimized path obtained by $\mathrm{A}^{*}$ algorithm of humanoid robot with whole modeling are shown in Figure 9.

The angular variation comparison diagram of $14 \mathrm{DOF}$ of the highly redundant mobile robot can be obtained by comparing the simulation results before and after motion optimization based on 2-norm, as shown in Figure 10. This robot includes 2 DOFs for the left and right wheels of the mobile platform, as shown in Figure 10(a) and 6 DOFs for the left and right arms of the robot, as shown in Figures 10(b) and 10(c), respectively.

Through the comparative analysis shown in Figure 10, the validity of motion optimization has been further proven by the simulation experiment of the dual-arm operating the optimized path of the highly redundant humanoid mobile robot with whole modeling.

Similarly, the robot is also divided into three parts to compare and analyze the MRSE value at each step in tracking different targets process, as shown in Figure 11. Through the analysis and comparison of the three robot parts, the conclusion is the same as the robot tracks the same target in subsection 3.1, and the validity of motion optimization has been further proven.

Each optimized RMSE value of the 14 DOFs is also generally lower than the non-optimized value during the dual-arm tracking optimized path processing. This result further proves the effectiveness of the motion optimization based on 2-norm from the perspective of energy reduction, as shown in Table 3.

Evidently, the motion by optimization based on 2-norm has slight fluctuation, improved smoothness, minimal energy consumption and considerably short path. The example is verified by the motion optimization based on 2-norm, and the results show that the motion optimization algorithm is effective in many aspects.

\section{Discussion and conclusions}

On the basis of whole modeling of a highly redundant humanoid mobile robot, this study conducted further research

Table 3 RMSE value comparison of the angular variations of the 14 DOFs in the dual -arm tracking the same optimized path by 3D a* heuristic algorithm before and after optimization

\begin{tabular}{|c|c|c|c|c|c|c|c|c|c|c|c|c|c|c|}
\hline \multirow[b]{2}{*}{14 DOFs } & \multicolumn{2}{|c|}{ Mobile platform } & \multicolumn{6}{|c|}{ Left arm } & \multicolumn{6}{|c|}{ Right arm } \\
\hline & Left wheel & Right wheel & 1 & 2 & 3 & 4 & 5 & 6 & 1 & 2 & 3 & 4 & 5 & 6 \\
\hline $\begin{array}{l}\text { RMSE values } \\
\text { RMSE values }\end{array}$ & 0.1275 & 0.1233 & 0.0881 & 0.0556 & 0.0153 & 0.0991 & 0.0668 & 0.1381 & 0.0802 & 0.0863 & 0.0916 & 0.1074 & 0.0956 & 0.0533 \\
\hline (Optimized) & 0.1133 & 0.0683 & 0.0664 & 0.0494 & 0.0498 & 0.0866 & 0.0825 & 0.1424 & 0.0577 & 0.0424 & 0.0308 & 0.0694 & 0.0380 & 0.0304 \\
\hline
\end{tabular}

Figure 12 Optimal path obtained by $\mathrm{A}^{*}$ algorithm tracking of robot

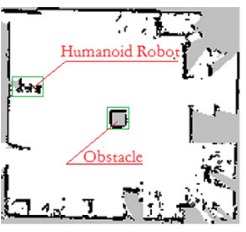

(a)

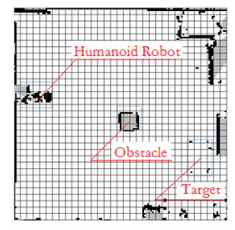

(b)

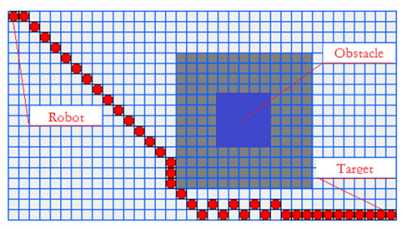

(c)

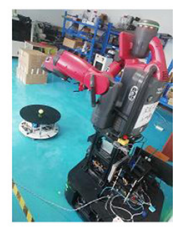

(d)

Notes: (a) Construction of environmental map; (b) Environment map rasterization; (c) Optimal path obtained by A* algorithm; (d) Robot tracking target 
on motion optimization based on the 2-norm of the aforementioned mobile robot. 2-norm (or Euclid Norm) is the linear distance between two vector matrices in space. We use the distance property of 2-norm to optimize the motion of a highly redundant humanoid mobile robot with whole modeling. The motion optimization algorithm has been verified by three different tracking and operating behaviors of the robot. Moreover, a comparison simulation is conducted between the before and after motion optimizations based on 2-norm. According to these simulations, the highly redundant humanoid mobile robot can completely track and operate different objects and can cooperate harmoniously when moving. According to the comparative study of each joint between the before and after motion optimizations, the motion optimization based on 2-norm can make the high redundant humanoid mobile robot's motion path considerably short, with minimal energy loss, and short time during the entire mobile tracking and operating process. The motion optimization algorithm is further verified by an example that this robot tracks an optimized path obtained by the $\mathrm{A}^{*}$ search algorithm. And some of the results have been achieved in the experiment, as shown in Figure 12.

In future studies, the proposed algorithms will be applied and further optimized, including the study of the singularity and dynamics on highly redundant humanoid mobile robots.

\section{References}

Baratcart, T. (2014), "Improved 2-norm-based redundancy resolution methods: with application to robotics", Master degree thesis, pp. 38-51.

Ge, L., Chen, J. and Li, R. (2011), "Research on kinematics of humanoid dual-arm for mobile robots", $\mathcal{F}$. Huazhong Univ. of Sci. E Tech. (Natural Science Edition), Vol. 39, pp. 1-4.

Han, G., Minglu, Z., Xiaojun, Z., Baijun, G. and Xiaochen, H. (2017), "Study on an approach to the solution of inverse kinematics for redundant manipulators", Modern Manufacturing Engineering, Vol. 8 No. 5, pp. 29-33.

Li, D., Wang, H. and You, B. (2009), "Path planning research and simulation of planar 3R redundant robot", The 2009 IEEE International Conference on Industrial Engineering and Applications, pp. 2855-2858.

Liu, C., Zhou, H. and Li, G. (2004), "Motion plan of mobile manipulator with differential driving", Fournal of Astronautics, Vol. 25 No. 2, pp. 183-186.
Nazari, V. and Notash, L. (2016), "Failure recovery of manipulators under joint velocity limits using constrained optimization and partitioned Jacobian matrix", Mechanism and Machine Theory, Vol. 99, pp. 58-71.

Petersson, L., Petersson, L. and Egerstedt, M. (2009), "Modeling of tracked mobile manipulators with consideration of track-terrain and vehicle-manipulator interactions", Robotics and Autonomous System, Vol. 57, pp. 1065-1074.

Sheng, J. (2017), "Research and application of industrial robot teaching technology based on gesture guidance", Master degree thesis, pp. 20-21.

Suryo Arifi, A. and Hang, M. (2013), "General framework of the force and compliant motion control for macro mini manipulator", IEEE/ASME International Conference on Advanced Intelligent Mechatronics (AIM), Vol. 1, pp. 949-954, Australia.

Vahrenkamp, N., Berenson, D., Asfour, T., Kuffner, J. and Dillmann, R. (2009), "Humanoid kinematic model for dualarm manipulation and re-grasping tasks", The 2009 IEEE/ RSf International Conference on Intelligent Robots and Systems, St. Louis, Vol. 1, pp. 2464-2470

Wang, W.Y. and Li, (2009), "RRT-based manipulation planning method for both arms of virtual human", fournal of System Simulation, Vol. 21 No. 20, pp. 6515-6518.

Wang, H., Li, R., Gao, Y., Cao, C. and Ge, L. (2016), "Comparative study on the redundancy of mobile single- and dual-arm robots", International fournal of Advanced Robotic Systems, Vol. 13 No. 6, pp. 1-19.

Xu, D., Hu, H., Calderon, C.A.A. and Tan, M. (2005), "Motion planning for a mobile manipulator with redundant DOFs", International fournal of InformationTechnology, Vol. 11 No. 11, pp. 1-10.

Zhang, T. and Ouyang, F. (2013), "Kinematics analysis and path planning of dual-robot coordinated couple motion", Fournal of Shanghai fiao Tong University, Vol. 47 No. 8, pp. 1251-1256.

Zhu, G., Chen, C., Tang, L. and Lin, D. (2007), "The realization and visualization of $3 \mathrm{D}$ shortest path analysis algorithm", Comuter Engineering and Applications, Vol. 43 No. 33, pp. 105-107.

\section{Corresponding author}

Ruifeng Li can be contacted at:1rf100@hit.edu.cn 